\title{
Las políticas en ciencia, innovación y tecnología y su relación con el contexto económico mexicano
}

Policies in science, innovation and technology

and its relationship with the Mexican economic context

\author{
Ana Laura Vargas Merino ${ }^{1}$ \\ Maricela Zúñiga Rodríguez ${ }^{2}$ \\ Universidad Autónoma del Estado de Hidalgo
}

Recibido: 14.12 .2020

Aceptado: 28.12.2020

\section{Resumen}

El presente documento es parte de la construcción de un estado del conocimiento de la investigación titulada: Condiciones para la investigación educativa en Ciencias de la Educación. Un estudio comparado entre universidades públicas en México. La revisión documental se basó en la técnica de Mapeamiento Informacional Bibliográfico (MIB) que permitió procesar fuentes bibliográficas (Molina, et al, 2013) y poder identificar categorías analíticas, para esta comunicación se presentan los resultados de la categoría: Las políticas en Ciencia, Innovación y Tecnología y su relación con el contexto económico mexicano. Este documento está conformado por tres ejes de análisis que le dan sentido: el primero está relacionado con la identificación de los acontecimientos en geopolítica; la relación entre economía del conocimiento y desarrollo de indicadores es el segundo eje de análisis; y el

\footnotetext{
1va267114@uaeh.edu.mx https://orcid.org/0000-0003-0305-6897

https://scholar.google.com/citations?hl=es\&authuser=2\&user=lzNoCQkAAAAJ

${ }^{2}$ maricela_zuniga@uaeh.edu.mx

https://orcid.org/0000-0002-8055-3742
} 
último eje de análisis es políticas en Ciencia, Innovación y Tecnología en México. Se finaliza con las conclusiones, las rutas de trabajo por desarrollar, y las referencias bibliográficas empleadas.

Palabras clave: Economía del conocimiento; Políticas públicas; Investigación.

\begin{abstract}
This document is part of the construction of a state of knowledge research entitled: Conditions for Educational Research in Educational Sciences. A comparative study among public universities in Mexico. The documentary review was based on the Bibliographic Information Mapping (MIB) technique that allowed to process bibliographic sources (Molina, et al, 2013) and to identify analytical categories, for this communication the results of the category are presented: Science, Innovation and Technology policies and their relationship with the Mexican economic context. This document is made up of three axes of analysis that give it meaning: the first is related to the identification of events in geopolitics; the relationship between the knowledge economy and the development of indicators is the second axis of analysis; and the last axis of analysis is policies in Science, Innovation and Technology in Mexico. It concludes with the conclusions, the work routes to develop, and the bibliographic references used.
\end{abstract}

Keywords: knowledge economy; Public policies; Research.

\title{
Introducción
}

El presente análisis: Las políticas en Ciencia, Innovación y Tecnología y su relación con el contexto económico mexicano, es parte de las primeras acciones para adentrarse en el tema de política pública e investigación, pero ¿Cómo dar ese primer paso sin perderse en un cúmulo de investigaciones? Se considera que es a través de la construcción de un estado del conocimiento, como una herramienta analítica y reconstructiva que ayude a identificar 
aquellos aspectos teóricos, situaciones problemáticas, metodologías y vacíos en el tema afín a este proyecto de investigación.

Se parte principalmente del Mapeamiento Informacional Bibliográfico (MIB) como una opción para localizar contenidos y procesar fuentes bibliográficas (Molina, et al, 2013), atendiendo rubros específicos que ayudan a determinar, desde una perspectiva sistémicodescriptiva, categorías de análisis, conceptos, tipos de fuentes e identificación de las ideas principales del autor.

En este caso, se concibe el contexto económico como marco para el desarrollo de políticas públicas en CIT (Ciencia, Innovación y Tecnología), siendo una de las categorías que se aborda en la investigación Condiciones para la investigación en Ciencias de la Educación. Estudio comparativo de universidades públicas en México. Para este documento se conformaron tres ejes de análisis que dan sentido: el primero está relacionado con la identificación de los acontecimientos en geopolítica; la relación entre economía del conocimiento y desarrollo de indicadores es el segundo eje de análisis; y el último eje de análisis es políticas en Ciencia, Innovación y Tecnología en México. Por último, se presentan las conclusiones y las rutas de trabajo por desarrollar.

\section{Ejes de análisis}

\section{Eje 1: Identificación de los acontecimientos en geopolítica}

Contexto económico y políticas de CIT (Ciencia, Innovación y Tecnología) es la primera categoría analítica que surge del procesamiento de 30 trabajos de investigación a través del MIB, implementada con de la revisión de informes internacionales, nacionales y notas periodísticas. Esta técnica ayudó a identificar el primer eje de análisis acontecimientos en geopolítica, pensando que son hechos que pueden relacionarse entre sí, como factores clave para la creación de indicadores de desarrollo y una agenda política internacional. Según la UNESCO (2015), a primera vista estos acontecimientos en geopolítica tienen poco que ver 
con la ciencia y la tecnología, sin embargo, a menudo han tenido una influencia indirecta significativa, que ha llevado a los gobiernos a estimular la inversión en I+D (Investigación y el Desarrollo), con respecto a su PIB (Producto Interior Bruto), perfilándose hacia una economía del conocimiento. Son diversos y pueden identificarse desde sucesos climáticos, movimientos sociales, conflictos bélicos, surgimiento de pandemias, cambios en la producción de mercado entre otros y de los cuales permanecerán sus efectos a futuro principalmente en el desarrollo de los países. Algunos de los más representativos son:

- Económicos: a) Creación de la $\mathrm{ASEAN}^{3}$, comunidad económica que tiene el objetivo de acelerar el crecimiento económico, fomentar la paz y la estabilidad en la región asiática; b) Recesión económica en $\mathrm{China}^{4}$, comprende una disminución en la producción industrial, manufacturera y energética que ha afectado a otras economías; c) creación del Brexit ${ }^{5}$ como retorno a una propia moneda del Reino Unido, que implica su salida de la Unión Europea; d) Firma del T-MEC ${ }^{6}$ : tratado de libre comercio entre México, Estados Unidos y Canadá, que cambiará las reglas de comercio entre estos países.

- Movimientos sociales: movimiento de los chalecos amarillos ${ }^{7}$ en Francia, ponen de manifiesto el descontento social con la clase gobernante, se replicó a otros países como Argentina y México.

\footnotetext{
${ }^{3}$ ASEAN (Asociación de Naciones del Sureste Asiático)-ONU. (2018, abril 1). Asociación de Naciones del Sudeste Asiático. Recuperado 20 de diciembre de 2019, de https://dppa.un.org/es/association-of-southeast-asiannations

${ }^{4}$ Arana, I. (2020, marzo 16). La economía china sufre más de lo esperado por el coronavirus. Recuperado 20 de mayo de 2020, de https://www.lavanguardia.com/economia/20200316/474193315948/china-economiacoronavirus-crisis-ventas-fabricas-empresas.html

${ }^{5}$ BBC News Mundo. (2020b, enero 31). Qué es el Brexit y otras 5 preguntas básicas para entender la salida de Reino Unido de la Unión Europea. Recuperado 27 de diciembre de 2019, de https://www.bbc.com/mundo/noticias-internacional-46521624

${ }^{6}$ BBC News Mundo. (2019, junio 19). T-MEC: 3 efectos en México del tratado de libre comercio que firmó con Estados Unidos y Canadá para reemplazar al TLCAN. Recuperado 17 de diciembre de 2019, de https://www.bbc.com/mundo/noticias-46404147

${ }^{7}$ Ayuso, S. (2020, marzo 14). Los 'chalecos amarillos' desafían al coronavirus y marchan en París. Recuperado 20 de mayo de 2020, de https://elpais.com/noticias/movimiento-chalecos-amarillos/
} 
- De salud: Pandemia de Covid $-19^{8}$ ha paralizado a las naciones, hasta el punto en algunos casos, de paralizar los sistemas sanitarios y afectar las economías de los países.

Siendo solo algunos ejemplos de acontecimientos geopolíticos, sin duda surgirán otros, que marcarán el futuro de las políticas internacionales y generarán impactos que influirán en el desarrollo de la CIT y en la producción científica. Al surgir como hechos independientes, no pueden ser controlados, pero sí deben ser considerados como parte de un sistema complejo y entrelazado para el desarrollo de los países.

Por ello es importante visualizar el desarrollo de la ciencia y la tecnología, desde la economía del conocimiento obliga, según Huissoud \& Gauchon, a "ubicar el contexto de geopolítica como referente, no solo de los cambios introducidos en la conformación de la geografía política como espacio y tiempo, sino en las estructuras de poder que definieron la formación y surgimiento de las relaciones de los Estados" (Muñoz Velasco \& Avedaño López, 2014). Pero también como análisis geopolítico, convirtiéndose en una herramienta de poder que ayuda a la definición del espacio, el entorno y la sociedad, que vaya más allá de la implementación de políticas científicas y tecnológicas, que orienten la acción investigativa en ciencia y tecnología para promover procesos transformativos en las estructuras de mercado y en la sociedad. Añaden Muñoz \& Avedaño (2014), que el Estado, recobra importancia para la acción gubernamental y para la comunidad científica, a su vez, asuma el liderazgo en el gasto en investigación y desarrollo (por ejemplo, en el aumento del porcentaje del Producto Interno Bruto -PIB- a ese rubro).

Sin embargo, esta visión del Estado líder en el rubro de la ciencia y la tecnología se contrapone con la concepción que Bourdieu (1992) tiene: el Estado no es un aparato orientado hacia el bien común, es un aparato de contención, de mantenimiento del orden público, pero en provecho de los dominantes; por lo que invita a pensar al Estado más allá de sus funciones económicas o ideológicas, siendo aspectos pendientes por analizar y desarrollar en este trabajo

\footnotetext{
${ }^{8}$ OMS (2020, abril 27). COVID-19: cronología de la actuación de la OMS. Recuperado 20 de mayo de 2020,
} de: https://www.who.int/es/news-room/detail/27-04-2020-who-timeline---covid-19 
de investigación. Trabajos de investigación siguen esta ruta crítica como los de Lemus Maciel (2018) que sostiene la idea sobre la incapacidad del Estado mexicano a través de las instituciones, planes de desarrollo, estrategias sectoriales y la política en ciencia y tecnología ha quedado manifiesta frente a un capitalismo polarizado que no ha permitido sentar las bases para un desarrollo pleno de fuerzas productivas en todos los sectores económico del país y muestra el carácter subordinado que tiene México ante los países desarrollados. Para Lemus Maciel (2018), el capitalismo no es homogéneo, aún en países desarrollados, pues se van a encontrar diferencias entre las formas y grados de desarrollo. En México el proceso de centralización del capital ha profundizado aún más las diferencias, entonces, ¿cómo encontrar relación entre el modelo económico internacional, frente a un país que presenta diferentes grados de desarrollo? ¿cómo relacionar la economía de un país con respecto a tendencias e indicadores de desarrollo? La respuesta al primer cuestionamiento puede ser desde la gestión de políticas públicas pertinentes y claras, que estén enfocadas a la realidad diversa que tiene este país. Con respecto al segundo cuestionamiento, se tratará en el siguiente eje de análisis.

\section{Eje 2: Economía del conocimiento y desarrollo de indicadores}

Se está viendo crecer la relación entre economía del conocimiento y desarrollo de indicadores, siendo un segundo aspecto identificado para esta categoría, lo que implica reflexionar la concepción de la sociedad del conocimiento junto con la idea de la economía del conocimiento, las cuales van moldeando a las instituciones hacia la cimentación de una institucionalidad centrada en tendencias en políticas públicas internacionales para la innovación y la creación de redes (Casalet, 2004); pero también pensar que esos indicadores que promueven una economía del conocimiento están marcando tendencias en las políticas de ciencia y tecnología a nivel mundial, las cuales se orientan hacia la innovación que permitan consolidar el crecimiento económico, la cohesión social y atender retos globales y sociales, como la pobreza, el cambio climático y la salud (Valero, Molina \& Ponce, 2019).

De acuerdo con la UNESCO (2015) la economía del conocimiento es la mejor forma de crear un motor de crecimiento eficaz que, de respaldo a los investigadores e inventores, por un lado, 
y por otro, los indicadores brindarán los caminos que los gobiernos pueden seguir. En la figura 2, se esquematizan los indicadores según cada organismo, los cuales van encaminados al desarrollo de una economía del conocimiento.

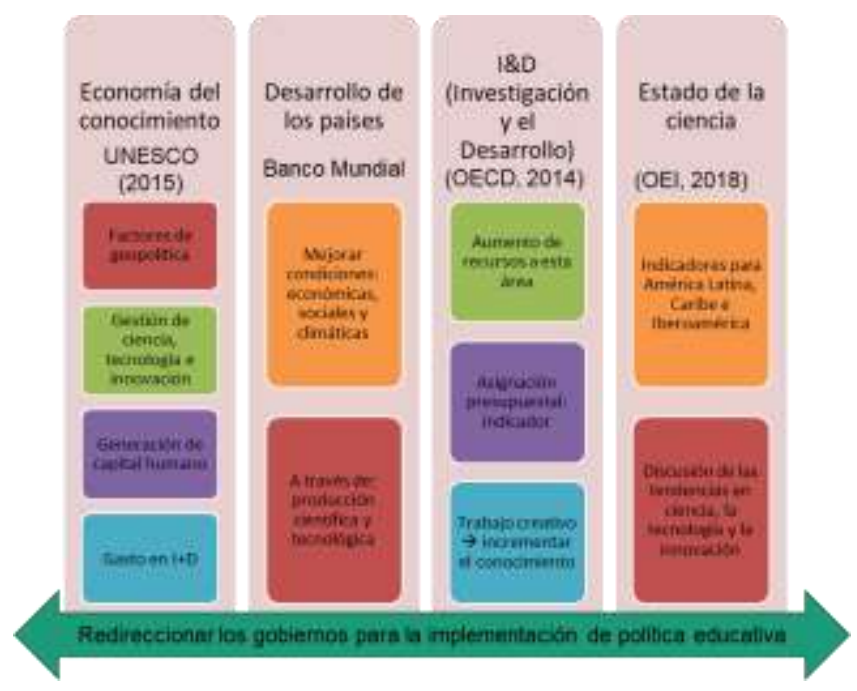

Figura 1. Economía y desarrollo de indicadores.

Creación propia inspirada en (UNESCO, 2015); (OCDE, 2018); (OEI, 2018);

Sin embargo, se observan tres problemáticas adicionales, la primera donde los países vean a los indicadores y tendencias como la receta mágica para mejorar sus situaciones económicas y sociales. En efecto, como menciona Parsons (2007), surgieron para la medición del cambio social y su auge se ha debido a la creencia de que el gobierno es el único que tiene la capacidad de resolver los problemas económicos y sociales. Por tanto, son solo apoyos para redireccionar a los gobiernos, desempeñan un papel en la toma de decisiones, principalmente en la creación de agenda pública en materia de ciencia y tecnología que impulse el desarrollo económico (Parsons, 2007), (Valero, Molina \& Ponce, 2019). Al respecto se suma que no todos los países tienen las mismas condiciones políticas, económicas y de desarrollo para poder promover una economía del conocimiento que permita contribuir a la investigación y desarrollo.

La segunda problemática identificada es hacia la medición del desarrollo de los países a través de los indicadores de ciencia y tecnología del Banco Mundial ${ }^{9}$, el cual se centra mayormente

\footnotetext{
${ }^{9}$ Banco Mundial. (2019, enero 1). Indicadores de ciencia y tecnología según el Banco Mundial. Recuperado 21 de mayo de 2020, de https://datos.bancomundial.org/indicador
} 
en la medición de investigación aplicada y experimental, debido a que la investigación básica (de corte teórico) no genera cálculos en los rubros de solicitudes de patentes, marca comercial y propiedad intelectual. Hecho que pone en jaque el apoyo económico para el desarrollo de investigaciones en el área de las humanidades, obligando a los investigadores de esta área a realizar investigaciones de frontera, que sean novedosas, creativas y sistemáticas, y a su vez que promuevan la transferencia de conocimiento y la certidumbre con respecto a costos y tiempo, además de contar con la característica de ser transdiciplinar, que se desarrolle en un sistema heterogéneo flexible y que incluya el trabajo compartido con la industria (sector privado) y el gobierno (OCDE, 2018).

La tercera problemática identificada es sobre el pobre financiamiento privado, que es $30 \%$ menor en México en comparación con medidas internacionales, que llega a ser superior al 50\% del gasto (CONACYT, 2017), (OEI, 2018), siendo un inconveniente para el país, para poder avanzar hacia una economía del conocimiento capaz de vincular al sector público con las universidades públicas, los centros de investigación y con el sector privado principalmente. Dicha desconexión muestra que, el sector privado empresarial tiene poco interés en apostar en la investigación del país, hecho que frena el desarrollo, como si ambos sectores privado y público estuvieran desconectados entre sí. Pero, la discusión no termina ahí, Pérez Mora \& Inguanzo (2018) invitan a reflexionar en torno al conocimiento, ya sea como bien de mercado o como un elemento democratizador y de inclusión social, siendo que, bajo el modelo basado en la economía del conocimiento se convierte más un bien para la transacción e intercambio económico, siendo un elemento sustancial para diversos actores, no solamente para la universidad o las comunidades científicas como las únicas interesadas en la producción, divulgación y uso de los conocimiento. Aunque Pérez Mora \& Inguanzo (2018) afirman que cada vez se otorga mayor importancia a la relación entre las universidades y las empresas, la realidad es que no es suficiente, pues se requiere de mayor participación, pues los últimos informes así lo afirman (ver OEI, 2018; OCDE, 2018; UNESCO, 2015). 


\section{Eje 3: Políticas en Ciencia, Innovación y Tecnología en México}

Entonces, si se reconoce la influencia de los acontecimientos en geopolítica, el desarrollo de una economía del conocimiento bajo indicadores y tendencias de desarrollo, además de la pobre participación del sector privado, ¿cómo hacer políticas en ciencia y tecnología pertinentes? El cuestionamiento lleva a desarrollar el último aspecto políticas en Ciencia, Innovación y Tecnología, que conforma esta categoría del estado del conocimiento. Se parte de los trabajos analíticos de Casalet (2004) que consisten en identificar cómo las políticas públicas contribuyen a movilizar las nuevas tendencias de mercado que se manifiestan a nivel sectorial y regional y constituyen la base de sustentación de la economía del conocimiento. La autora demuestra que:

Entonces si se reconoce la influencia de los acontecimientos en geopolítica, el desarrollo de una economía del conocimiento bajo indicadores y tendencias de desarrollo, además de la pobre participación del sector privado, ¿cómo hacer políticas en ciencia y tecnología pertinentes? El cuestionamiento lleva a desarrollar el último aspecto políticas en Ciencia, Innovación y Tecnología, que conforma esta categoría del estado del conocimiento. Se parte de los trabajos analíticos de Casalet, que consisten en identificar cómo las políticas públicas contribuyen a movilizar las nuevas tendencias de mercado que se manifiestan a nivel sectorial y regional y constituyen la base de sustentación de la economía del conocimiento. La autora demuestra que:

...a medida que aumenta el contenido científico y tecnológico de la actividad económica, se reorganiza la acción y gestión institucional para sostener las nuevas capacidades de aprendizaje de las empresas, así como de los nuevos agentes que desde diferentes ámbitos (públicos, privados, académicos) contribuyen a desarrollar la diversidad de las capacidades de la sociedad. (Casalet, 2004, p. 62)

Autores como Gómez Collado (2017) llevan sus reflexiones en torno a la política educativa actual en México, la cual describen como mиy compleja, porque, por un lado, comprende la 
acción educadora sistemática que el Estado lleva a cabo por medio de las instituciones educativas y por el otro, la acción que realiza al guiar, orientar o modificar la vida cultural de todo el país. Lo que lleva a pensar sobre el marco político en educación, ciencia y tecnología, principalmente en la agenda pública nacional, como el área de acción de políticas (Aguilar, 1992), del cual se identifica que: 1) el Plan Nacional de Desarrollo 2019-2024, menciona que el CONACYT coordinará un plan en beneficio de la sociedad y del desarrollo nacional con la participación de universidades, pueblos, científicos y empresas (DOF, 2019), sin embargo, a dos años y medio de gobierno, no hay más información que determine cómo se llevará a cabo y cómo incidirá en el gasto de la CIT en México, incluso, no se menciona si se continuará con el desarrollo de una economía del conocimiento.

A este panorama se suma que aún no está lista la Ley General de Humanidades, Ciencias, Tecnologías e Innovación 2020. Se pretende hacer una consulta ciudadana y de instituciones para la elaboración de esta ley, sin embargo, hay incertidumbre entre la comunidad científica del país. La incertidumbre va en aumento, (no solo por la pandemia de salud por el Covid-19), ya que en reiteradas circunstancias se habla de la llamada austeridad republicana ${ }^{10}$, que se traduce como el recorte de presupuesto público a organismos que dependen del gobierno federal, a la fecha se ha solicitado reducir el gasto hasta en un 50\% en servicios generales y gastos de operación. En caso específico el CONACYT ${ }^{11}$, principal organismo público que asesora al gobierno para articular políticas públicas y promover la investigación científica, ha sufrido un recorte presupuestario que se traduce en una fiscalización de los recursos, replanteándose o extinguiendo fondos.

Por último, se puede sumar que se ha identificado una desconexión con respecto a los organismos científicos que acreditan, legitiman, reconocen y validan a los que producen ciencia en este país, generando tención entre la generación de investigación individual con

\footnotetext{
${ }^{10}$ AMLO refuerza austeridad republicana, pide más recorte al gasto de gobierno. (09 de abril de 2020). Forbes México. Recuperado de https://www.forbes.com.mx/amlo-refuerza-austeridad-republicana-pide-mas-recorte-agasto-de-gobierno/

${ }^{11}$ Tras recorte presupuestal, CONACYT hará más con menos: Álvarez-Buylla. (05 de febrero de2019) Aristegui noticias. Recuperado de https://aristeguinoticias.com/0502/mexico/tras-recorte-presupuestal-conacyt-hara-mascon-menos-alvarez-buylla/
} 
respecto a la producción colectiva en relación con la producción individual (Pérez Mora \& Naidorf, 2015). Afirmación que podemos constatar al revisar los programas que regulan y generan lineamientos y mecanismos para que los investigadores puedan acceder a estímulos económicos y reconocimientos por su producción científica (Ponce Crespo, 2017) como son Sistema Nacional de Investigadores ${ }^{12}$ (SNI) y el Programa para el Desarrollo Profesional Docente $(\mathrm{PRODEP})^{13}$, se ha identificado que los procesos pueden ser distintos o contradictorios entre sí, por lo que el investigador debe realizar dos procesos diferentes, privilegiando y sosteniendo la burocratización de la ciencia.

Toda esta reflexión nos muestra que, el campo político, no solo como el espacio de acción de las políticas como lo plantea Aguilar (1992), sino como Bourdieu (2000) lo concibe, es una arena de combates, enfrentamientos declarados y que permite a su vez, construir de manera rigurosa esta realidad que es la política y su juego político, para así compararla con otras realidades, por ejemplo, el campo académico y de investigación. A su vez, ayuda a definir rutas pendientes como el análisis del marco político mexicano sobre CIT, considerando el gobierno actual, la agenda pública y su relación con los programas que regulan la investigación en México (SNI y PRODEP).

\section{Conclusiones}

La revisión documental ha permitido identificar que el mundo es un sistema interconectado entre sí, por lo que estudiar algún aspecto específico, ya sea la ciencia, la innovación y la tecnología, implica ver la conexión que puede guardar con los acontecimientos en geopolítica, considerando que tienen una influencia indirecta y que sus efectos podrán permanecer a futuro, ya que son hechos independientes que no pueden ser controlados. Partir de esta visión de mundo, deja como ruta, pensar en el Análisis de los Sistema Mundo de Immanuel

\footnotetext{
${ }^{12}$ CONACYT. (2019, diciembre 28). Sistema Nacional de Investigadores. Recuperado 21 de mayo de 2020, de https://www.conacyt.gob.mx/index.php/el-conacyt/sistema-nacional-de-investigadores

${ }^{13}$ SEP. (s. f.). Programa para el Desarrollo Profesional Docente, para el Tipo Superior (PRODEP). Recuperado 28 de diciembre de 2019, de http://www.dgesu.ses.sep.gob.mx/PRODEP.htm
} 
Wallerstein (Aguirre Rojas, 2007), principalmente desde la perspectiva del análisis de los sistemas-mundo en la historia inmediata y de futuros escenarios.

Entonces el contexto de la geopolítica como espacio tiempo y como estructuras de poder brinda referentes con respecto a las relaciones de Estado, pero también como herramienta orienta la formulación de políticas para promover procesos transformativos en las estructuras de mercado y en la sociedad. Aspecto lleva a reflexionar sobre el papel actual de los Estados y la dicotomía que se presenta con respecto al tipo de economía que las naciones pretenden desarrollar. Según la revisión documental, la economía del conocimiento no siempre proviene de un capitalismo homogéneo, todo lo contrario, son más evidentes las diferencias económicas, entonces ¿qué papel juegan los indicadores y tendencias de desarrollo? Ciertamente la economía del conocimiento y el desarrollo van de la mano, pero se enfrenta a diversas problemáticas, por ejemplo, el pobre financiamiento privado, que es $30 \%$ menor en México en comparación con otros países que llega a ser superior al 50\% del gasto, siendo el principal inconveniente, para poder transcitar hacia una economía de ese estilo.

Por tanto, las políticas en CIT en México, se desarrollan bajo un marco geopolítico complejo que se enfrente a la insertidumbre que deja el recorte presupuestario, la austeridad en los organismos y programas que sirven de mediación para la investigación. A este panorama se suma: la desconexión entre los programas que regulan la investigación, ya que cada uno tiene sus propios procesos y regulaciones (SNI y PRODEP), que deja como ruta de trabajo el análisis estratégico de sus políticas, como marco contextual y de desarrollo. 


\section{Referencias}

Aguilar, L. (1992). Estudio introductorio. En L. Aguilar, El estudio de las políticas públicas (págs. 15-35). México: Grupo editorial Miguel Ángel Porrúa.

Aguirre Rojas, C. A. (2007). Immanuel Wallerstein y la perspectiva crítica del Análisis de los Sistema-Mundo. Textos de economía, 10(2). Recuperado de https://periodicos.ufsc.br/index.php/economia/article/view/1850/1613

Bourdieu, P. (1992). Sobre el Estado. Cursos en el Collège de France (1989-1992). Barcelona: Editorial Anagrama.

Bourdieu, P. (2000). Sobre el Campo Político. Francia: Presses Universitaires de Lyon.

Casalet Ravenna, M. (2004). Construcción institucional del mercado en la economía del conocimiento. Revista UNAM, 1(002), 52-63. Recuperado de http://revistas.unam.mx/index.php/ecu/article/view/2803/2363

CONACYT. (2017). Informe General del estado de la ciencia, la tecnología y la innovación 2017. Ciudad de México: Consejo Nacional de Ciencia y Tecnología. Recuperado de http://www.siicyt.gob.mx/index.php/transparencia/informes-conacyt/informe-general-delestado-de-la-ciencia-tecnologia-e-innovacion/informe-general-2017

DOF. (2019). Plan Nacional de Desarrollo 2019-2014. Ciudad de México. Recuperado de: dof.gob.mx/nota_detalle.php?codigo=5565599\&fecha=12/07/2019

Gómez Collado, M. E. (2017). Panorama del sistema educativo mexicano desde la perspectiva de las políticas públicas. Innovación Educativa, 17(74), 143-163. Recuperado de http://www.scielo.org.mx/pdf/ie/v17n74/1665-2673-ie-17-74-00143.pdf 
Lemus Maciel, B. (2018). Políticas en Ciencia y Tecnología en México 2006-2017. (Tésis inédita de Doctorado). Universidad Nacional Autónoma de México.

Molina, A., Pérez, R., Bustos, E., Castaño, C., Jardey, O., \& Sánchez, M. (2013). Mapeamiento Informacional Bibliográfico de Enfoques y Campos Temáticos de la diversidad cultural: el caso de las revistas CSSE, Sci Edu. And Sci \& Edu. IX Encontro Nacional de Pesquisa em Educação em Ciências.

Muñoz Velasco, L. A., \& Avedaño López, J. (2014). Geopolítica, ciencia y tecnología en las sociedades con instituciones políticas y mercados no inclusivos. Revista Administración \& Desarrollo, 43(59), 41-51. 4 Recuperado de https://www.researchgate.net/publication/318219078_Geopolitica_ciencia_y_tecnologia_en _las_sociedades_con_instituciones_politicas_y_mercados_no_inclusivos OCDE. (2018). Guía para la recopilación y presentación de información sobre la investigación y el desarrollo experimental. Fundación Española para la Ciencia y la Tecnología. Recuperado de https://www.oecd.org/publications/manual-de-frascati-2015-9789264310681-es.htm

OEI. (2018). El estado de la ciencia. Principales indicadores de Ciencias y tecnología iberoamericanos e interamericanos. Buenos Aires: Altuna Impresores S.R.L. Recuperado de http://www.ricyt.org/wp-content/uploads/2018/10/www.ricyt_.org_files_edlc_2018.pdf

Parsons, W. (2007). Políticas públicas. Una introducción a la teoría y la práctica del análisis de políticas. En W. Parsons, Análisis de las decisiones (págs. 273-476). EE.UU: Miño y Dávila.

Pérez Mora, R., \& Inguanzo Arias, B. (2018). La movilización del conocimiento en las políticas científicas en México. Horizontes Sociológicos, 69-81. Recuperado de https://www.researchgate.net/publication/326450487_La_movilizacion_del_conocimiento_e n_las_politicas_cientificas_en_Mexico 
Ponce Crespo, C. I. (2017). Las identidades científicas en la Universidad Autónoma del Estado de Hidalgo. Un análisis comparativo entre dos institutos. (Tesis de inédita de Doctorado). Universidad Nacional Autónoma de México, México.

UNESCO. (2015). Informe de la UNESCO sobre la ciencia. Hacia el 2030. París: Organización de las Naciones Unidas para la Educación. Recuperado de https://unesdoc.unesco.org/ark:/48223/pf0000235407_spa

Valero Olmedo, C., Molina García, A., \& Ponce Crespo, C. I. (2019). Políticas de ciencia, tecnología e innovación en México: aproximación a su análisis. Edähi Boletín Científico de Ciencias Sociales y Humanidades del ICSHu, 8(15), 65-72. Recuperado de https://repository.uaeh.edu.mx/revistas/index.php/icshu/article/view/5256/6855 
REVISTA INTERNACIONAL DE PEDAGOGÍA E INNOVACIÓN EDUCATIVA |

Volumen 1. Número 1. Enero - Junio 2021

ISSN: 2745-0341 (En línea) 